\title{
Intracarotid cold saline infusion contributes to neuroprotection in MCAO-induced ischemic stroke in rats via serum and glucocorticoid-regulated kinase 1
}

\author{
DAZHI WANG ${ }^{1,2 *}$, ZHI HUANG ${ }^{3 *}$, LEI LI $^{4}$, YINGNAN YUAN ${ }^{5}$, LEI XIANG ${ }^{5}$, \\ XIAOWEN WU ${ }^{5}$, CAIFANG NI ${ }^{1}$ and WENFENG $\mathrm{YU}^{2}$ \\ ${ }^{1}$ Department of Interventional Radiology, The First Affiliated Hospital of Soochow University, Suzhou, Jiangsu 215006; \\ ${ }^{2}$ Key Laboratory of Molecular Biology, Guizhou Medical University, Guiyang, Guizhou 550002; \\ ${ }^{3}$ Department of Interventional Radiology, The Second Affiliated Hospital of Guizhou Medical University, Kaili, \\ Guizhou 556000; ${ }^{4}$ Department of General Courses, People's Armed College of Guizhou University, Guiyang, \\ Guizhou 550025; ${ }^{5}$ School of Medical Imaging, Guizhou Medical University, Guiyang, Guizhou 550002, P.R. China
}

Received December 20, 2018; Accepted July 16, 2019

DOI: $10.3892 / \mathrm{mmr} .2019 .10599$

\begin{abstract}
Intracarotid cold saline infusion (ICSI) brings about neuroprotective effects in ischemic stroke. However, the involvement of serum and glucocorticoid-regulated kinase 1 (SGK1) in the underlying mechanism of ICSI is not fully understood; therefore, we used the rat middle cerebral artery occlusion (MCAO) model to investigate the neuroprotective effects of ICSI on ischemic stroke in rats, as well as the involvement of SGK1 in these effects. ICSI decreased infarct size and brain swelling, as determined by 2,3,5-triphenyltetrazolium chloride staining and the dry-wet weight method, respectively. The results of terminal deoxynucleotidyl transferase mediated nick end labeling (TUNEL) and Nissl staining showed that ICSI also suppressed apoptosis and increased the relative integral optical density (IOD) values of Nissl bodies in the rat MCAO model. Regarding the mechanism, the results of immunohistochemistry and western blotting revealed that ICSI upregulated SGK1 expression and downregulated beclin-1 and LC-3 expression in the rat MCAO model. In addition, SGK1 knockdown increased ICSI-mediated infarct size and brain
\end{abstract}

Correspondence to: Dr Caifang Ni, Department of Interventional Radiology, The First Affiliated Hospital of Soochow University, 889 Pinghai Road, Suzhou, Jiangsu 215006, P.R. China

E-mail: fangcaini66@outlook.com

Dr Wenfeng Yu, Key Laboratory of Molecular Biology, Guizhou Medical University, 9 Beijing Road, Guiyang, Guizhou 550002, P.R. China

E-mail: 2291860147@qq.com

${ }^{*}$ Contributed equally

Key words: hypothermia, cerebral ischemia, infarct, intracarotid cold saline infusion, autophagy, serum and glucocorticoid-regulated kinase 1 swelling, promoted apoptosis, and reduced the IOD values of Nissl bodies in the rat MCAO model. In addition, we found that SGK1 knockdown upregulated beclin-1 and LC-3 expression mediated by ICSI. Overall, ICSI had a neuroprotective effect on ischemic stroke after reperfusion by upregulating SGK1 and inhibiting autophagy.

\section{Introduction}

Therapeutic hypothermia is an effective method of neuroprotection (1) that is currently used to treat diseases such as hypoxic ischemic encephalopathy and severe craniocerebral trauma caused by cardiac arrest, with good results (2). Selective brain cooling (SBC) is a therapeutic, low-temperature method that has no effect on whole-body temperature (3). Since SBC avoids the side effects of low temperature, it has received increasing clinical and research attention. In particular, intracarotid cold saline infusion (ICSI) can cool the brain rapidly and uniformly; it is therefore considered an ideal SBC method (4). ICSI is the fastest and most effective way to lower brain temperature and protect the brain (5), with its protective effect being mediated through vascular flushing and low temperature $(6,7)$. In previous studies, ICSI administered before reperfusion improved brain function after middle cerebral artery occlusion (MCAO) (8). The protective effect of ICSI on the brain results from hypothermia and cerebral artery flushing (5). However, the underlying mechanism of ICSI in the brain of patients with ischemic stroke is unclear.

The serum and glucocorticoid-regulated kinase 1 (SGKI) gene encodes a serine/threonine protein kinase that plays an important role in the cellular stress response (9). This kinase activates certain chloride, sodium and potassium channels, suggesting that it is involved in the regulation of cell survival, renal sodium excretion, and neuronal excitability (10). High expression of this gene may be associated with the development of several diseases, including hypertension and diabetic nephropathy (11). SGK1 is a member of the AGC protein kinase family and plays an important role in regulating ion 
channel expression, as well as in promoting proliferation and survival in malignant epithelial cells (12). Recently, several studies have confirmed that SGK1 has a protective effect on ischemia-reperfusion injury. For instance, one investigation reported alterations in hippocampal $S G K 1$ gene expression in rats with transient cerebral ischemia (13). Furthermore, SGK1 was recently shown to play a protective role in the hippocampal neurons of rats with ischemia-reperfusion injury (14). In another study, the corticotropin-induced estrogen enhancement effect of SGK1 expression protected the heart from ischemia-reperfusion injury (15). However, no investigations have yet explored whether ICSI can affect SGK1 expression.

Autophagy is a cellular pathway that regulates the transportation of cytoplasmic macromolecules and organelles to lysosomes for degradation (16). Beclin-1 and microtubule-associated protein 1 light chain 3 (LC-3) are two important proteins involved in autophagic flux (17-19). Previous studies have shown that activation of autophagy plays an important role in cerebral ischemia and reperfusion injury $(20,21)$. However, it remains unclear whether autophagic flux is related to the neuroprotective effects of ICSI and SGK-1.

In the present study, we investigated the role of ICSI and clarified the mechanism of its neuroprotective effect.

\section{Materials and methods}

Animals. In total, 250 male 8 week old Sprague-Dawley rats weighing $280 \mathrm{~g}$ were acquired from The First Affiliated Hospital of Soochow University (Jiangsu, China). The rats housed at a temperature of $22-25^{\circ} \mathrm{C}$ and $50-65 \%$ humidity, with a $12 \mathrm{~h}$ light/dark cycle and ad libitum access to food and water. The rats were raised individually in an animal facility. All procedures involving animals were approved by the Animal Research Committee of The First Affiliated Hospital of Soochow University.

MCAO model establishment. To conduct surgery, the rats were anesthetized using an intraperitoneal injection of $10 \%$ chloral hydrate (400 mg/kg; cat. no. C8383, Sigma-Aldrich/Merck $\mathrm{KGaA}$ ). Peritonitis was monitored and not observed during the study. Two temperature probes (Physitemp, Clifton, NJ, USA) were placed in the right cerebral cortex $(3 \mathrm{~mm}$ lateral to bregma, $3 \mathrm{~mm}$ posterior, and $4 \mathrm{~mm}$ under the surface of the skull), as well as in the rectum, to continuously monitor temperature. An improved intraluminal filament was used to induce MCAO. After $2 \mathrm{~h}$, reperfusion was established by filament shrinkage. To confirm the success rate of MCAO surgery and reperfusion, blood flow in the right cortex of the right MCA was measured using a laser Doppler flow meter $2 \mathrm{~mm}$ in the posterior fontanelle and $6 \mathrm{~mm}$ in the anterior fontanelle. After the filaments were inserted, $30 \%$ of the rats without blood flow reduction were lower than baseline. Reperfusion success was defined as an $80 \%$ increase over the baseline in blood flow after the filament was contracted. In groups treated with ICSI, the filament was contracted, and a modified PE50 canaliculus was inserted into the internal carotid artery about $5 \mathrm{~mm}$ from the external carotid artery. Next, by infusion with freshly made $10^{\circ} \mathrm{C}$ saline at a rate of $10-25 \mathrm{ml} / \mathrm{h}$, the brain temperature was reduced to $33^{\circ} \mathrm{C}$ for $20 \mathrm{~min}$. Normal infusion groups received intraperitoneal normal saline infusion (INSI) at $37^{\circ} \mathrm{C}$, while the other procedures were identical to those in the ICSI groups. A non-infusion group underwent insertion of a filtration membrane to a depth of $15-\mathrm{mm}$ insufficient to induce MCAO and no infusion of renal tubules. After transfusion, the rats were subjected to rapid rewarming using a heating pad and bubble wrap; when their brain temperature had returned to baseline, they were considered reheated.

Grouping. The rats were randomly divided into five groups: i) non-infusion group (MCAO rats with no infusion; $n=25$; mortality rate: $4.0 \%$ ); ii) ICSI group (MCAO rats treated with ICSI immediately after reperfusion; $n=90$; mortality rate: $15.6 \%$ ); iii) INSI group (MCAO rats treated with INSI immediately after reperfusion; $\mathrm{n}=25$; mortality rate: $8.0 \%$ ); iv) ICSI+reperfusion group (MCAO rats treated with ICSI for $1 \mathrm{~h}$ immediately after reperfusion; $\mathrm{n}=85$; mortality rate: $11.8 \%$ ); and v) INSI+reperfusion group (MCAO rats treated with INSI for $1 \mathrm{~h}$ immediately after reperfusion; $\mathrm{n}=25$; mortality rate: $8.0 \%$ ). In addition, shRNAs for SGK1, as well as negative control (NC) shRNAs, were obtained from GenePharma Co., Ltd. (Shanghai, China). Rats in the ICSI and ICSI+reperfusion groups were treated using NC shRNAs and SGK1 shRNAs, respectively, while those in the ICSI group $(n=50)$ were divided into the ICSI+NC subgroup $(n=25$; mortality rate: $4.0 \%)$ and ICSI+shSGK1 subgroup $(n=25$; mortality rate: $6.0 \%$ ); the rats in the ICSI+reperfusion group $(n=50)$ were divided into the ICSI+reperfusion+NC subgroup $(n=25$; mortality rate: $6.0 \%)$ and ICSI+reperfusion+shSGK1 subgroup ( $n=25$, the mortality rate: $6.0 \%$ ). After treatment for $50 \mathrm{~h}$, the indicators were evaluated, the rats were euthanized, and their tissues were used in subsequent experiments. During the course of the experiment, the animals were also euthanized by cervical dislocation when they reached the standard of the humane endpoint. The mortality rate represents animals prematurely euthanized after reaching humane endpoints, rather than animals that were found dead. Humane endpoint is a refinement strategy to reduce the pain and suffering suffered by the animals during the experiment as much as possible. Humane endpoints mainly include weight loss of 20-25\%, loss of appetite (anorexia more than $24 \mathrm{~h}$ ), inability to stand, severe infection, tumors more than $10 \%$ of body weight, organ system failure, dyspnea, severe anemia, renal failure and peritoneal fluid. Animals were euthanized when two or more humane endpoints were observed.

Infarct size. As described in previous studies $(22,23)$, after reperfusion for $48 \mathrm{~h}$, the animals were anesthetized by intraperitoneal injection of $10 \%$ chloral hydrate $(400 \mathrm{mg} / \mathrm{kg})$ and then decapitated. The brain was rapidly removed, and the coronal section of brain was sliced in a special groove (thickness: $2 \mathrm{~mm}$ ) after cooling in ice-cold saline for $10 \mathrm{~min}$. The slices were immediately immersed in $4 \mathrm{ml}$ of $1 \%$ 2,3,5-triphenyltetrazolium chloride (TTC) and incubated at $37^{\circ} \mathrm{C}$ for $10 \mathrm{~min}$. Next, the TTC solution was replaced with $4 \%$ polyoxymethylene. After $8 \mathrm{~h}$, a digital camera was used to photograph the sections, and the infarct size was measured using the UTHSCSA Image Tool 3.0 software (https://uthscsa-imagetool.software.informer.com/). To eliminate the effect of postoperative edema on injury volume, the infarcted volume (\%) was calculated according to the following method: 
(Volume of the left hemisphere-Non-infarct volume of right hemisphere)/(Volume of the left hemisphere) x100\%.

Brain swelling. According to previous research (24), brain swelling was assessed using a 0.35-T Signa Ovation Excite MRI scanner (General Electric, Milwaukee, WI, USA).

Knockdown of SGK1. For SGK1 knockdown, SGK1 and control shRNA were acquired from RiboBio (Guangzhou, Guangdong, China). SGK1 knockdown rats were obtained from the Shanghai Research Center for Model Organisms (Shanghai, China). In brief, after anesthesia, rats were placed on a stereotactic apparatus, and $50 \mu \mathrm{g} S G K 1$ and control shRNA vectors were injected into the right ischemia region of rats, and the final volume was $10 \mu \mathrm{l}$. The sequence of shSGK1 was as follows: Top strand, 5'-CACCGCCGGCTGTGCCTT CTCTCCATTCAAGAGATGGAGAGAAGGCACAGCCG GC-3'; bottom strand, 5'-AAAAGCCGGCTGTGCCTT CTCTCCATCTCTTGAATGGAGAGAAGGCACAGCCG GC-3'; Ds Oligo, 5'-CACCGCCTTCTCTCCATCCGC TGCTTTCAAGAGAAGCAGCGGATGGAGAGAAGGC-3' and 3'-CGGAAGAGAGGTAGGCGACGAAAGTTC TCTTCGTCGCCTACCTCTCTTCCGAAAA-5'.

Terminal deoxynucleotidyl transferase mediated nick end labeling (TUNEL) staining. To identify apoptosis in the ipsilateral hippocampus, TUNEL staining was performed using the In Situ Cell Death Detection Kit (Roche, Indianapolis, IN, USA) according to the manufacturer's instructions. Briefly, the sections were washed for $30 \mathrm{~min}$. They were then incubated in $0.1 \%$ Triton X-100 and $0.1 \%$ sodium citrate and rinsed three times using phosphate-buffered saline (PBS) for $10 \mathrm{~min}$ each time. The slices were then incubated in $0.3 \% \mathrm{PBS} / 0.1 \%$ Tween-20 for $30 \mathrm{~min}$ to inhibit endogenous peroxidase activity; they were then once again rinsed in PBS. Next, the sections and the 50- $\mu 1$ TUNEL reaction mixture of the in situ Cell Death Detection Kit were incubated in a moist atmosphere for $60 \mathrm{~min}$ at $37^{\circ} \mathrm{C}$ in the dark. The slices were rinsed three times in PBS and observed under a Nikon TE300 microscope (Nikon, Inc., Tokyo, Japan). A negative control was achieved in each pore by incubating the slices in $50-\mu 1$ labeled solution instead of TUNEL reaction mixture. At least 10 randomly selected fields were used to count TUNEL-positive cells (x200 magnification). The cell count was performed by researchers who had been blinded to the rat conditions.

Nissl staining. Three days after the operation, the rats were anesthetized using $10 \%$ chloral hydrate $(400 \mathrm{mg} / \mathrm{kg}$ body weight) by intraperitoneal injection, and their brains were extracted and fixed in $4 \%$ paraformaldehyde for $2 \mathrm{~h}$. After continuous overnight incubation in $0.1 \mathrm{~mol} / 1 \mathrm{PBS}$ containing 20 and $25 \%$ glucose, the brain was cut into $10-\mathrm{mm}$ coronal sections using a cryogenic thermostat (Leica CM1950; Leica, Wetzlar, Germany) and stored at $20^{\circ} \mathrm{C}$. Then, Nissl staining solution (Beyotime Institute of Biotechnology, Shanghai, China) was used, following the manufacturer's instructions. Briefly, the sections were soaked in $1 \%$ toluidine blue and dyed for $5 \mathrm{~min}$ at $50^{\circ} \mathrm{C}$, washed with double distilled water, and then dehydrated with gradient ethanol; the sections were observed under a Nikon Eclipse E400 microscope (x200 magnification; Nikon, Inc.).

Immunohistochemical analysis. Frozen brain sections in OCT compound (14- $\mu \mathrm{m}$ thick) were formalin-fixed and subjected to immunohistochemical analysis to determine SGK1 expression. Endogenous peroxidase was blocked using 3\% $\mathrm{H}_{2} \mathrm{O}_{2}$ for $5 \mathrm{~min}$. The sections were then washed in PBS for a short time and cooled at room temperature for $20 \mathrm{~min}$. They were then rinsed again in PBS. Non-specific protein was blocked by incubation in $5 \%$ horse serum for $40 \mathrm{~min}$. The sections were incubated with primary antibodies (anti-SGK1; diluted 1:200; cat. no. YB-33275M; Suzhou Ard Biological Co., Ltd., Shanghai, China) for $1 \mathrm{~h}$ at room temperature. They were then incubated with fluorescein isothiocyanate/cyanine 3-conjugated IgG (1:500 dilution; cat. no. SC-2340; Santa Cruz Biotechnology, Inc., Santa Cruz, CA, USA) at room temperature for $60 \mathrm{~min}$. Ten microscopic fields per section were randomly photographed (x200; Nikon TE300; Nikon, Inc.) to allow the SGK-1-positive cells to be counted.

Western blotting. Treated tissues were lysed using RIPA buffer (Sigma-Aldrich/Merck KGaA). Total protein was separated using 10\% SDS-PAGE and transferred to a polyvinylidene fluoride membrane (cat. no. 88518; Thermo Fisher Scientific, Inc., Waltham, MA, USA). The membranes were blocked with $5 \%$ non-fat milk for $1 \mathrm{~h}$ at room temperature and then subjected to incubation at $4{ }^{\circ} \mathrm{C}$ overnight in primary antibodies against SGK1 (1:500; cat. no. ab59337), beclin-1 (1:2,000; cat. no. ab207612;), LC-3 (1:3,000; cat. no. ab51520) and $\operatorname{GAPDH}(1: 2,500 ;$ cat. no. ab9485; all Abcam, Cambridge, UK). The next day, after washing, the membranes were incubated in secondary, peroxidase-conjugated anti-mouse $(1: 2,000$, cat. No. 7076; Cell signaling Technology, lnc.) or anti-rabbit (1:2,000, cat. No. 5127; Cell signaling Technology, lnc.) antibodies for $1.5 \mathrm{~h}$ at room temperature. Finally, the results were determined using an enhanced chemiluminescence (ECL) substrate kit in an ECL system (Amersham Pharmacia). Quantity One software version 4.6.2 (Bio-Rad Laboratories, Inc., Hercules, CA, USA) was used for densitometric analysis.

Statistical data analysis. All data are presented as mean \pm standard error, and Graphpad Prism 6 (GraphPad Software, Inc., La Jolla, CA, USA) was used for statistical analysis. Student's t-test was used to evaluate the difference between two groups; one-way ANOVA with Tukey's post hoc was applied to assess the differences among more than two groups, and P-values $<0.05$ were considered as indicative of statistical significance.

\section{Results}

ICSI alleviates infarct size and brain swelling after MCAO. To induce ischemic stroke, MCAO was performed in Sprague-Dawley rats for $2 \mathrm{~h}$ by applying an intraluminal filament. The MCAO rats were treated with either ICSI or INSI after reperfusion (Fig. 1A). After reperfusion for $48 \mathrm{~h}$, the infarct size was determined using TTC staining; brain swelling was also measured. The results indicated that the infarct size was significantly reduced in the ICSI and INSI groups compared with the non-infusion group; ICSI also alleviated 
A
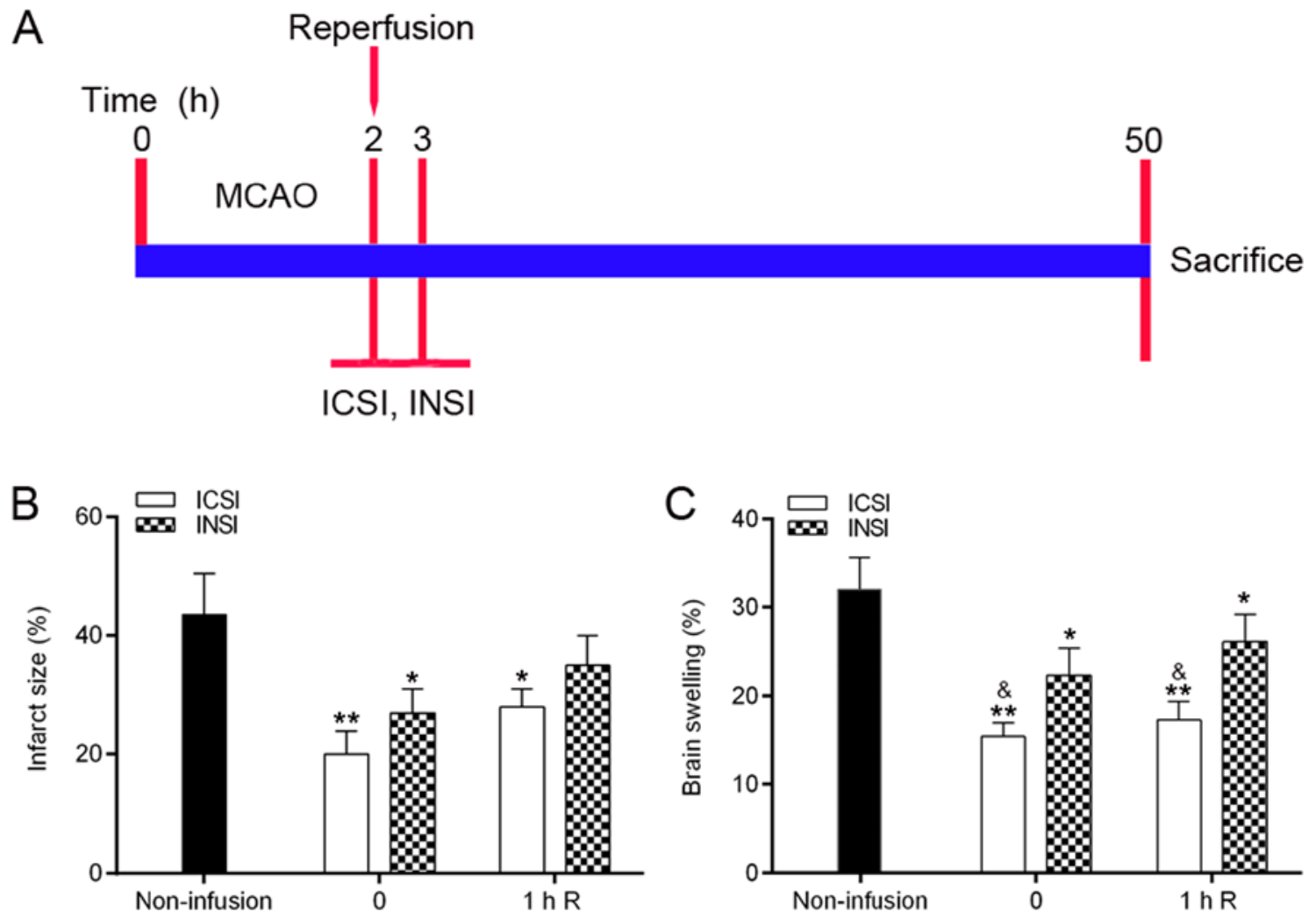

Figure 1. ICSI decreases infarct size and brain swelling in the rat MCAO model. Rats were randomly divided into the MCAO group (n=24), ICSI group $(n=26)$, INSI group $(n=23)$, ICSI+reperfusion group $(n=25)$, and INSI+reperfusion group $(n=23)$. (A) Experimental procedure in each group of rats is shown. (B) Infarct size and (C) brain swelling were detected in the rat MCAO models treated with reperfusion and either ICSI or INSI. " $\mathrm{P}<0.05,{ }^{* *} \mathrm{P}<0.01$ (infusion groups vs. non-infusion group); ${ }^{\circledR} \mathrm{P}<0.05$, ICSI vs. INSI. One-way ANOVA with Tukey's post hoc test was performed. All experiments were repeated three times. MCAO, middle cerebral artery occlusion; ICSI, intracarotid cold saline infusion; INSI, intracarotid normothermic saline infusion. 0 , immediate infusion after reperfusion; $1 \mathrm{~h} \mathrm{R}, 1 \mathrm{~h}$ after reperfusion.

the infarct size after reperfusion in the MCAO model $(\mathrm{P}<0.05$, $\mathrm{P}<0.01$; Fig. 1B). Brain swelling was significantly decreased in the ICSI and INSI groups relative to the non-infusion group. After reperfusion for $1 \mathrm{~h}$, the brain swelling in the ICSI and INSI groups also showed significant reductions. In addition, brain swelling was significantly decreased in the ICSI group compared with the INSI group after reperfusion in the MCAO model $(\mathrm{P}<0.05, \mathrm{P}<0.01$; Fig. 1C).

ICSI alleviates cell apoptosis and nerve injury in the brain. To further explore the effects of ICSI on apoptosis and nerve injury in the brain after reperfusion in the MCAO model, TUNEL and Nissl staining were performed. There were significantly fewer TUNEL-positive cells in the ICSI and ICSI-1h-R groups than that noted in the non-infusion, INSI and INSI-1h groups $(\mathrm{P}<0.05, \mathrm{P}<0.01, \mathrm{P}<0.001$; Fig. $2 \mathrm{~A}$ and $\mathrm{B})$. We also found that the relative integral optical density (IOD) values of Nissl bodies were higher in the ICSI and ICSI-1h-R groups than in the non-infusion group, that they were higher in the INSI and INSI-1h groups than in the non-infusion group, and that they were higher in the ICSI and ICSI-1h-R groups than in the INSI and INSI- 1 h groups $(\mathrm{P}<0.01, \mathrm{P}<0.001$; Fig. $2 \mathrm{~A}$ and $\mathrm{C})$.

ICSI increases the expression of SGKI and decreases the expression of the autophagy markers beclin-1 and LC-3. To determine the expression of SGK1, immunohistochemistry was performed. The results showed that SGK1 expression was higher in the infusion groups than that in the non-infusion group, that it was higher in the ICSI group than in the INSI group, and that it was higher in the ICSI-1h-R group than in the INSI-1h-R group (Fig. 3A). In addition, we confirmed that SGK1 expression was higher in the infusion groups than in the non-infusion group, that it was significantly higher in the ICSI and ICSI-1h-R groups than in the INSI and INSI-1h-R groups. The expression of both beclin-1 and LC-3 was significantly downregulated in the infusion groups compared with that in the non-infusion group, and it was significantly decreased in the ICSI and ICSI-1h-R groups compared with that in the INSI and INSI-1h-R groups $(\mathrm{P}<0.05, \mathrm{P}<0.01, \mathrm{P}<0.001$; Fig. 3B).

Knockdown of SGKI decreases the protective effect of ICSI. To investigate whether ICSI contributes to SGK1-mediated neuroprotection in rats with ischemic stroke, animals in the ICSI and ICSI-1h-R groups were transfected with SGK1 shRNA. Four groups of rats were then subjected to cold saline infusion: Rats given immediate ICSI (ICSI group), SGK1-knockdown rats given immediate ICSI (ICSI+shSGK1), rats given ICSI starting $1 \mathrm{~h}$ after reperfusion (ICSI-1h-R), and SGK1-knockdown rats given ICSI starting $1 \mathrm{~h}$ after reperfusion (ICSI-1h-R+shSGK1; Fig. 4A). A western blot assay was used to verify the knockdown effect of SGK1, and the results revealed that SGK1 expression was significantly suppressed after transfection with SGK1 shRNAs $(\mathrm{P}<0.01, \mathrm{P}<0.001$; Fig. 4B). TTC staining and the dry-wet weight method were 

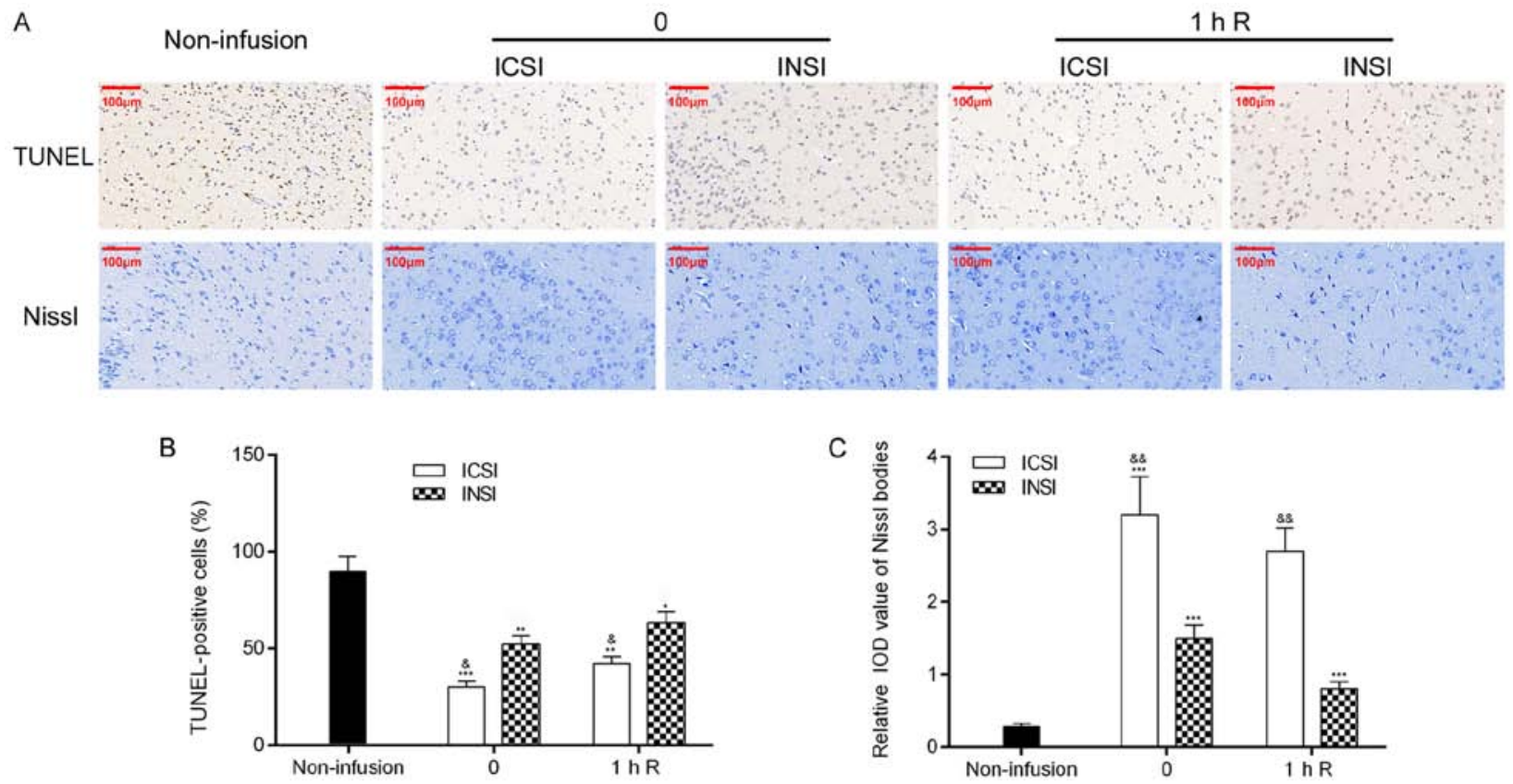

Figure 2. ICSI inhibits apoptosis and increases the relative IOD value of Nissl bodies in the rat MCAO model. (A) Apoptosis and the relative IOD value of Nissl bodies were measured using TUNEL staining and Nissl staining, respectively. Magnification, x100; scale bar, $100 \mu \mathrm{m}$. (B) TUNEL-positive cells were analyzed. (C) Relative IOD value of Nissl bodies was determined. ${ }^{*} \mathrm{P}<0.05,{ }^{* *} \mathrm{P}<0.01,{ }^{* * *} \mathrm{P}<0.001$, infusion groups vs. non-infusion group. ${ }^{\text {\& }} \mathrm{P}<0.05$, ${ }^{\text {\& }} \mathrm{P}<0.01$, ICSI vs. INSI group; comparisons were carried out at the same time point. Data were evaluated using one-way ANOVA with Tukey's post hoc test. All experiments were repeated three times. IOD, integral optical density; MCAO, middle cerebral artery occlusion; ICSI, intracarotid cold saline infusion; INSI, intracarotid normothermic saline infusion. 0, immediate infusion after reperfusion; $1 \mathrm{~h} \mathrm{R}, 1 \mathrm{~h}$ after reperfusion.

A

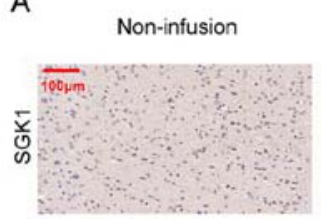

0

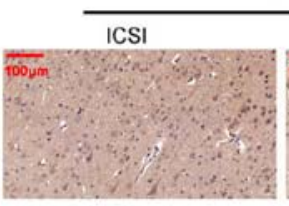

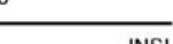

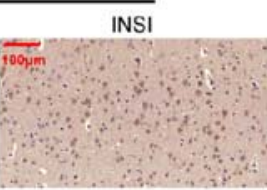

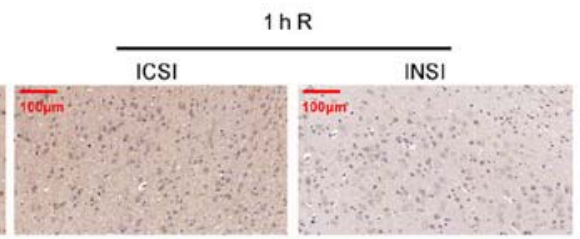

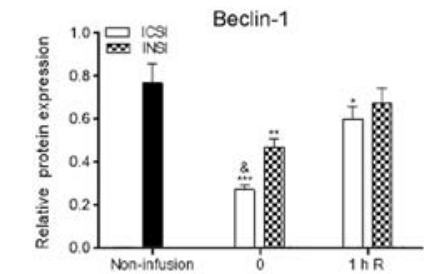

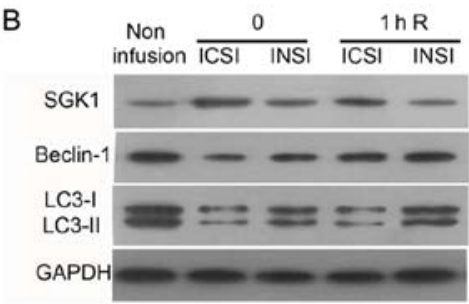
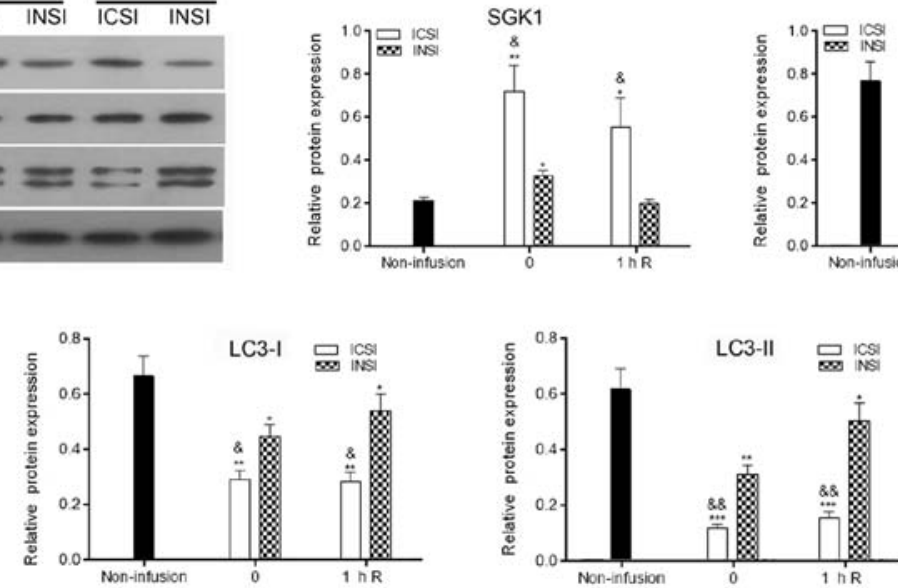

Figure 3. ICSI upregulates SGK1 expression and downregulates beclin-1 and LC-3 expression in the rat MCAO model. (A) SGK1 expression was assessed using immunohistochemistry. Magnification, $\mathrm{x} 100$; scale bar, $100 \mu \mathrm{m}$. (B) Western blot analysis of SGK1, beclin-1 and LC-3 expression. "P<0.05, **P $<0.01$, ${ }^{* * *} \mathrm{P}<0.001$, infusion groups vs. non-infusion group; ${ }^{\circledR} \mathrm{P}<0.05,{ }^{\&} \mathrm{P}<0.01$, ICSI vs. INSI; comparisons were carried out using one-way ANOVA with Tukey's post hoc test at the same time point. All experiments were repeated three times. SGK1, serum and glucocorticoid-regulated kinase 1; MCAO, middle cerebral artery occlusion; ICSI, intracarotid cold saline infusion; INSI, intracarotid normothermic saline infusion. 0 , immediate infusion after reperfusion; $1 \mathrm{~h} \mathrm{R}, 1 \mathrm{~h}$ after reperfusion.

applied to measure infarct size and brain swelling. The results revealed that knockdown of SGK1 using shRNAs significantly increased infarct size and brain swelling, as compared to the
ICSI group $(\mathrm{P}<0.05, \mathrm{P}<0.01$; Fig. $4 \mathrm{C}$ and $\mathrm{D})$. Therefore, we suggest that SGK1 knockdown aggravated the process of ischemic stroke treatment using ICSI. 
A

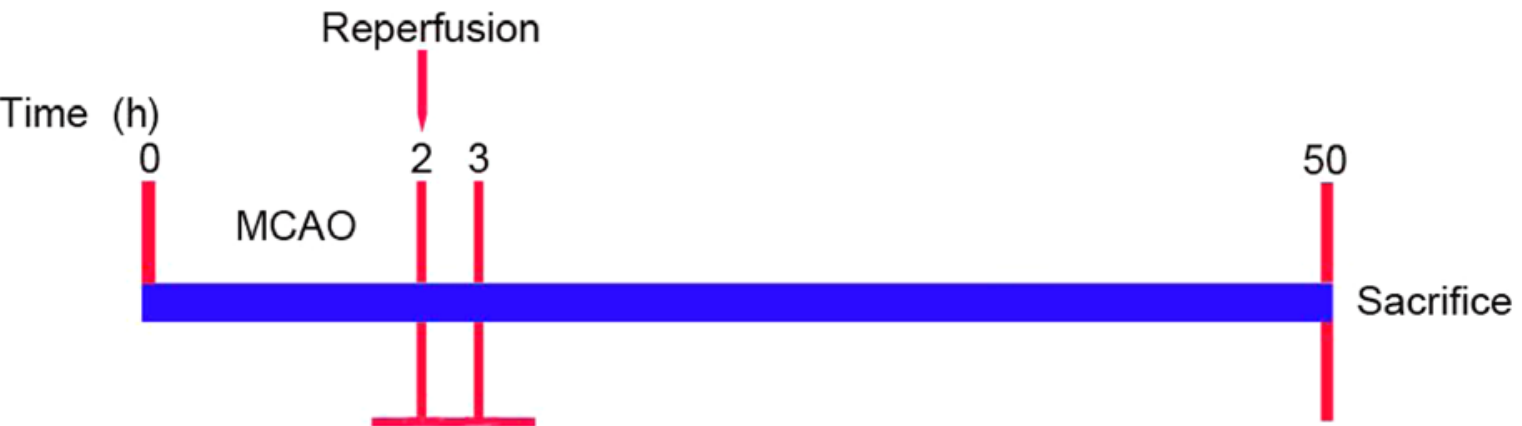

ICSI, INSI, shSGK1

B
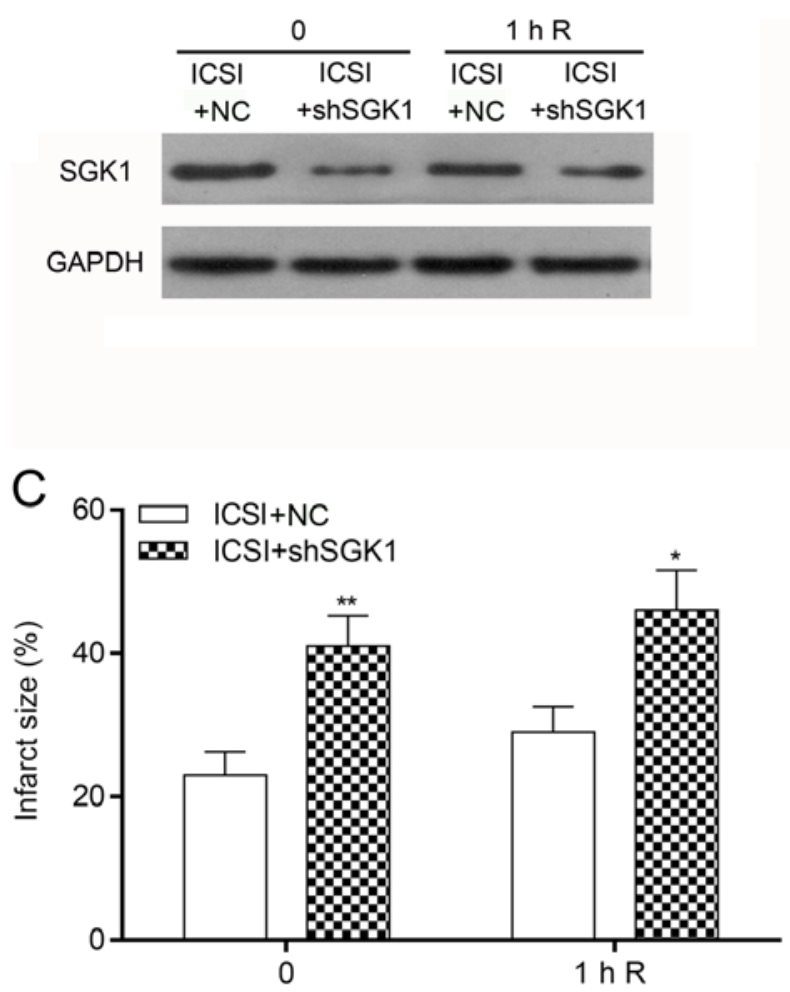

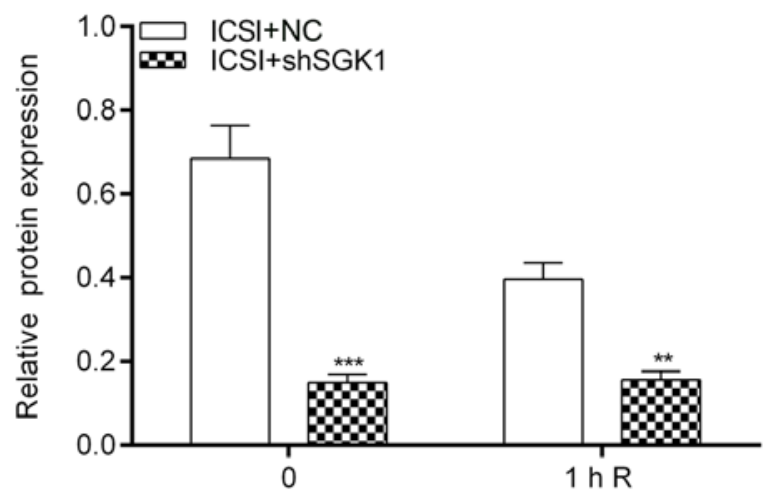

D

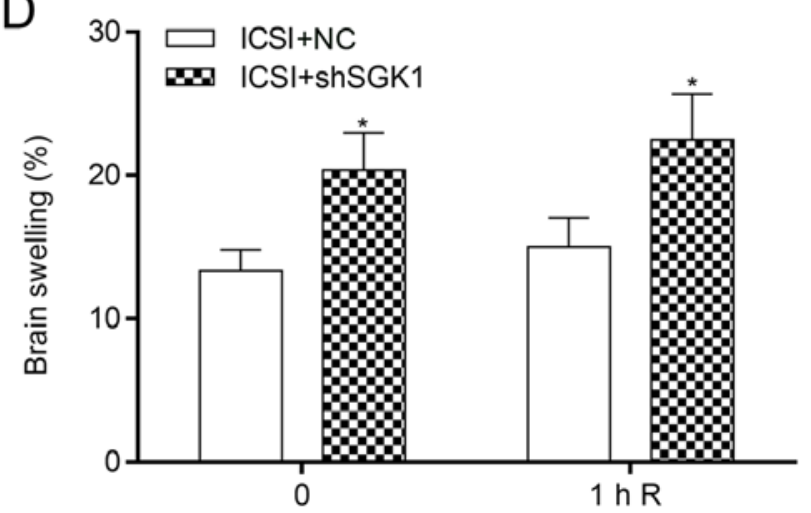

Figure 4. SGK1 knockdown increases infarct size and brain swelling mediated by ICSI. NC shRNAs or SGK1 shRNAs were transfected into the rats in the ICSI and ICSI+reperfusion groups. The rats were divided into the ICSI+NC group $(n=24), I C S I+s h S G K 1$ group $(n=23)$, ICSI+reperfusion+NC group $(n=23)$, and ICSI+reperfusion+shSGK1 group ( $\mathrm{n}=23$ ). (A) Protocol of the experiments. (B) SGK1 expression was examined by western blot analysis in each group. (C) Infarct size was measured using TTC staining after SGK1 knockdown in the ICSI group. (D) The brain swelling of the ICSI group after SGK1 knockdown. ${ }^{*} \mathrm{P}<0.05,{ }^{* * *} \mathrm{P}<0.01,{ }^{* * * *} \mathrm{P}<0.001$ vs. ICSI group by Student's t-test. All experiments were repeated three times. SGK1, serum and glucocorticoid-regulated kinase 1; MCAO, middle cerebral artery occlusion; ICSI, intracarotid cold saline infusion; INSI, intracarotid normothermic saline infusion. 0 , immediate infusion after reperfusion; $1 \mathrm{~h} \mathrm{R}, 1 \mathrm{~h}$ after reperfusion.

Knockdown of SGK1 decreases the protection of neurons from apoptosis and injury. To explore whether SGK1 affects apoptosis and ischemic stroke injury in rats treated using ICSI, TUNEL staining and Nissl staining were performed. The results showed that SGK1 knockdown using shRNAs promoted ICSI-mediated apoptosis in the MCAO model $(\mathrm{P}<0.05, \mathrm{P}<0.01 ;$ Fig. 5A and $\mathrm{B})$. Meanwhile, we demonstrated that SGK1 knockdown significantly increased the relative IOD value of Nissl bodies mediated by ICSI in the MCAO model $(\mathrm{P}<0.01$, Fig. 5A and $\mathrm{C})$. Therefore, we confirmed that SGK1 knockdown significantly accelerated apoptosis and increased the relative IOD value of ICSI-mediated Nissl bodies in the rat MCAO model.
Knockdown of SGKI upregulates ICSI-mediated downregulation of beclin-1 and LC-3 expression. Furthermore, western blotting was performed to analyze the influence of SGK1 knockdown on the expression of beclin-1 and LC-3 downregulated by ICSI in the rat MCAO model. These results indicated that the ICSI-mediated decreases in the expression of Beclin1 and LC3 could be significantly upregulated by the knockdown of SGK-1 ( $\mathrm{P}<0.05, \mathrm{P}<0.01, \mathrm{P}<0.001$; Fig. 6).

\section{Discussion}

Ischemic stroke involves hemiplegia and disturbance of consciousness caused by cerebral thrombosis, cerebral 
A
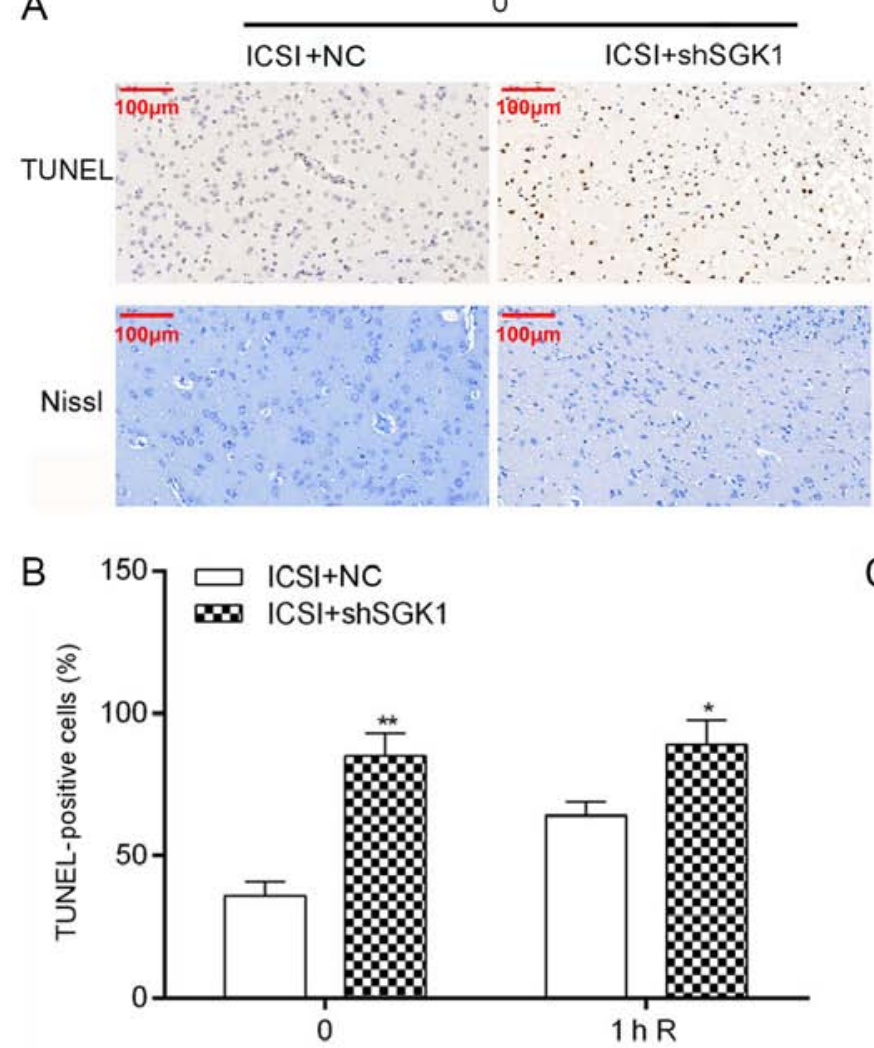

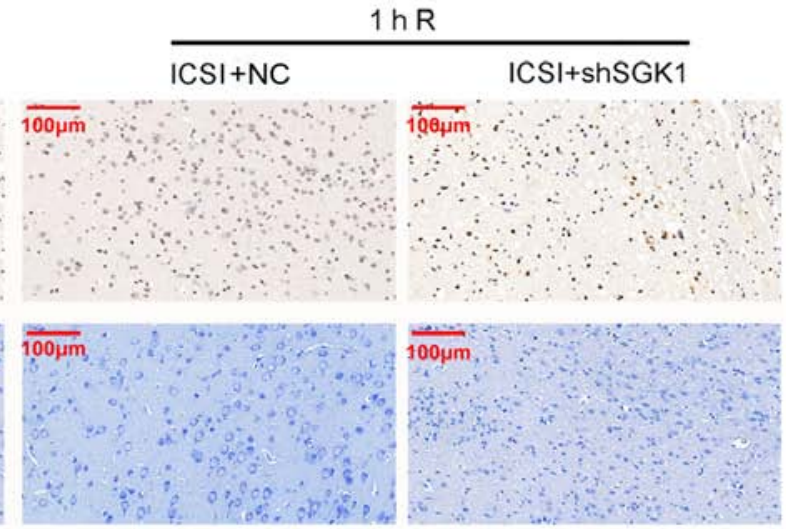

C

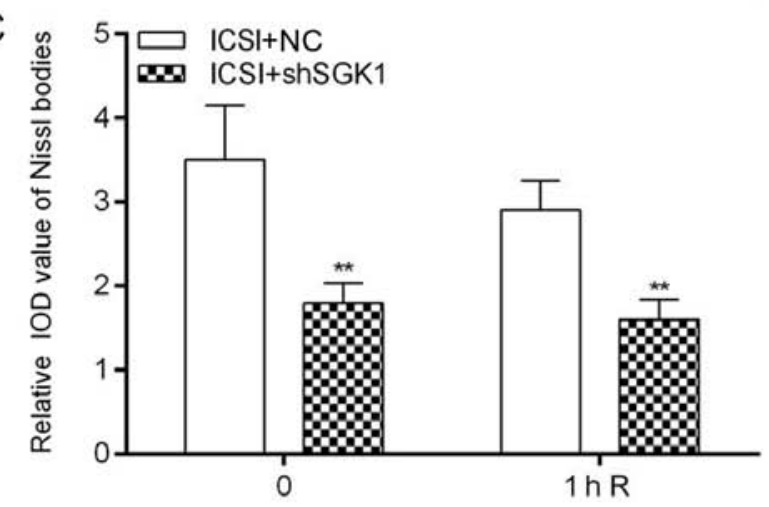

Figure 5. SGK1 knockdown promotes apoptosis and decreases the related IOD values in ICSI-mediated Nissl bodies in the rat MCAO model. (A) TUNEL staining and Nissl staining in the ICSI group after SGK1 knockdown (shSGK1). Magnification, x100; scale bar, $100 \mu \mathrm{m}$. (B) TUNEL staining in the ICSI groups after SGK1 knockdown. (C) Nissl staining in the ICSI groups after SGK1 knockdown. ${ }^{*} \mathrm{P}<0.05,{ }^{* *} \mathrm{P}<0.01$ vs. ICSI+NC group by Student's t-test. All experiments were repeated three times. SGK1, serum and glucocorticoid-regulated kinase 1; MCAO, middle cerebral artery occlusion; ICSI, intracarotid cold saline infusion; 0 , immediate infusion after reperfusion; $1 \mathrm{~h} \mathrm{R}, 1 \mathrm{~h}$ after reperfusion.
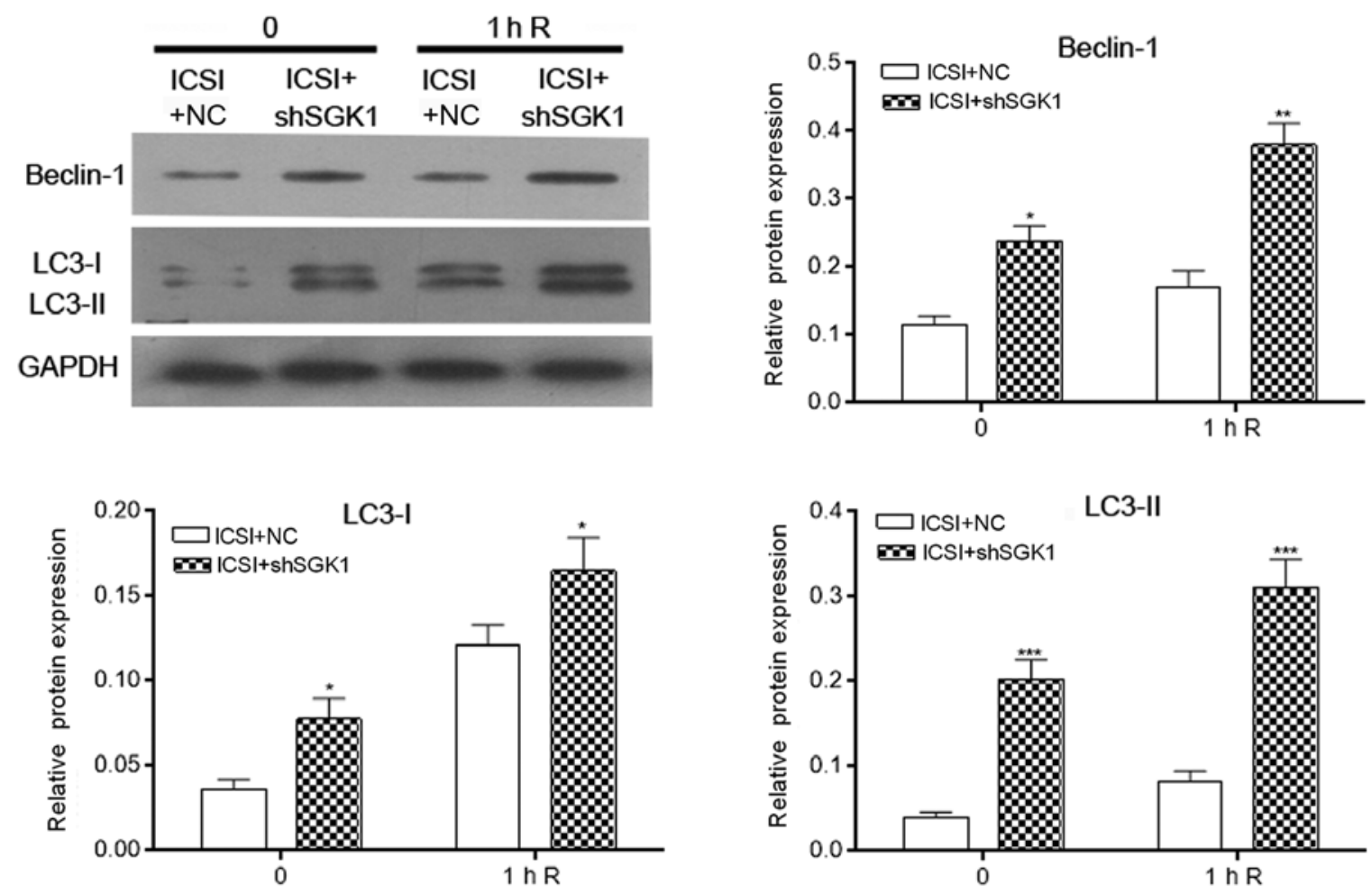

Figure 6. SGK1 knockdown upregulates ICSI-mediated decreased beclin-1 and LC-3 expression in the rat MCAO model. Western blot analysis of beclin-1 and LC-3 in the ICSI groups after SGK1 knockdown. ${ }^{*} \mathrm{P}<0.05,{ }^{* * *} \mathrm{P}<0.01,{ }^{* * *} \mathrm{P}<0.001$ vs. ICSI+NC group by Student's t-test. All experiments were repeated three times. SGK1, serum and glucocorticoid-regulated kinase 1; MCAO, middle cerebral artery occlusion; ICSI, intracarotid cold saline infusion; 0, immediate infusion after reperfusion; $1 \mathrm{~h} \mathrm{R}, 1 \mathrm{~h}$ after reperfusion. 
artery occlusion, and consequent cerebral infarction (25). At present, ischemic stroke is the most common cerebrovascular disease (26). It is characterized by its high incidence, high disability rate, high recurrence rate, and high fatality rate, and it has become a major threat to human health (27). Therefore, it is crucial that researchers and clinicians discover preventative and treatment strategies.

Therapeutic hypothermia is an effective method of protecting brain cells, and it is widely used to treat hypoxic-ischemic encephalopathy (28). The neuroprotective mechanisms of low temperature include i) reducing the metabolism of nerve cells and reducing acidosis; ii) inhibiting the production and release of endogenous harmful substances, such as glutamic acid, aspartic acid and serotonin; iii) protecting the blood-brain barrier and reducing cerebral edema and intracranial pressure; iv) preventing the proliferation of polymorphonuclear leukocytes and reducing the intracellular inflammatory response; v) altering the transmission of genetic information and promoting protein synthesis and recovery; and vi) inhibiting nerve cell apoptosis (29). Intracarotid cold saline infusion (ICSI) is a novel and selective cryogenic method (30,31). A large number of clinical studies have shown that ICSI can rapidly reduce brain temperature in rats, significantly protecting brain function and ensuring clinical safety $(25,32,33)$.

In the present study, the rat middle cerebral artery occlusion (MCAO) model was established and treated using ICSI and intracarotid normothermic saline infusion (INSI). TTC staining and brain swelling measurement were used to evaluate the effects of ICSI on local brain tissues. In the present study, ICSI was found to alleviate infarct size and brain swelling in the MCAO model after reperfusion. In addition, it was confirmed that ICSI inhibited cell apoptosis and nerve injury in the brain. Therefore, it was further demonstrated that ICSI is a fast, efficient, and safe method of implementing selective hypothermia in the brain. Selective cerebral hypothermia during acute ischemia has therapeutic effects on ischemic reperfusion, including reducing infarct volume, reducing brain swelling, and improving neurological deficits.

Previous research has revealed that serum and glucocorticoid-regulated kinase 1 (SGK1) protects against ischemia-reperfusion injury. For instance, dexamethasone, which is associated with SGK1, can protect against renal ischemia-reperfusion injury (34). SGK1 is involved in renal ischemia-reperfusion injury (35), while glucocorticoid affects SGK1 expression and protects against ischemia-reperfusion injury after heart transplantation (36). SGK1 inhibits cell death and inflammation in the ischemic-reperfused heart (37). In the present study, it was demonstrated that ICSI increased SGK1 expression and that SGK1 silencing increased infarct size and brain swelling, accelerated apoptosis, and decreased the relative IOD value of ICSI-mediated Nissl bodies. Therefore, we suggest that ICSI protected against brain injury in MCAO-induced ischemic stroke rats.

Autophagy is an evolutionarily conserved process in eukaryotes that processes the turnover of intracellular substances $(16,38,39)$. Many studies have shown that maladjusted autophagy regulation is related to various diseases, such as malignant tumors, autoimmune disease, neurodegenerative disease and pathogenic microorganism infection (40-43). Beclin-1 and LC-3 serve as specific markers of autophagy and have been applied in both clinical and basic research $(44,45)$. In the present study, it was revealed that ICSI downregulated beclin-1 and LC-3 expression and that knockdown of SGK1 upregulated beclin-1 and LC-3 expression. ICSI inhibited autophagy by regulating SGK1 expression in the rat MCAO model.

ICSI was demonstrated to have neuroprotective potential in MCAO-induced ischemic stroke. In addition, ICSI significantly upregulated SGK1 expression, and SGK1 silencing promoted cerebral injury in MCAO-induced ischemic stroke. Furthermore, it was confirmed that ICSI was conducive to the neuroprotective efficacy of MCAO-induced ischemic stroke in rats by SGK1 and autophagy. However, additional studies are needed to validate the function of SGK1 and the potential mechanism of ICSI in ischemic stroke, and we must optimize our experimental grouping.

\section{Acknowledgements}

Not applicable.

\section{Funding}

The present study was supported by the National Science Foundation (grant. nos. 81860321and 81460276).

\section{Availability of data and materials}

The datasets used during the present study are available from the corresponding author upon reasonable request.

\section{Authors' contributions}

DW, ZH, CN and WY conceived and designed the study. DW and $\mathrm{ZH}$ performed the experiments. LL, YY, LX and XW analyzed the data and were the primary contributors to the writing of the manuscript. All authors read and approved the final manuscript.

\section{Ethics approval and consent to participate}

The present study was approved by the Ethics Committee of The First Affiliated Hospital of Soochow University.

\section{Patient consent for publication}

Not applicable.

\section{Competing interests}

The authors declare that they have no competing interests.

\section{References}

1. González-Ibarra FP, Varon J and López-Meza EG: Therapeutic hypothermia: Critical review of the molecular mechanisms of action. Front Neurol 2: 4, 2011.

2. Froehler MT and Ovbiagele B: Therapeutic hypothermia for acute ischemic stroke. Expert Rev Cardiovasc Ther 8: 593-603, 2010.

3. Choi JH, Marshall RS, Neimark MA, Konstas AA, Lin E, Chiang YT, Mast H, Rundek T, Mohr JP and Pile-Spellman J: Selective brain cooling with endovascular intracarotid infusion of cold saline: A pilot feasibility study. AJNR Am J Neuroradiol 31: 928-934, 2010. 
4. Luan X, Li J, McAllister JP II, Diaz FG, Clark JC, Fessler RD and Ding Y: Regional brain cooling induced by vascular saline infusion into ischemic territory reduces brain inflammation in stroke. Acta Neuropathol 107: 227-234, 2004

5. Ji Y, Hu Y, Wu Y, Ji Z, Song W, Wang S and Pan S: Therapeutic time window of hypothermia is broader than cerebral artery flushing in carotid saline infusion after transient focal ischemic stroke in rats. Neurol Res 34: 657-663, 2012.

6. Cheng H, Ji X, Ding Y, Luo Y, Wang G, Sun X, Chen J and Ling F: Focal perfusion of circulating cooled blood reduces the infarction volume and improves neurological outcome in middle cerebral artery occlusion. Neurol Res 31: 340-345, 2009.

7. Chen J, Ji X, Ding Y, Luo Y, Cheng H and Ling F: A novel approach to reduce hemorrhagic transformation after interventional management of acute stroke: Catheter-based selective hypothermia. Med Hypotheses 72: 62-63, 2009.

8. Linares G and Mayer SA: Hypothermia for the treatment of ischemic and hemorrhagic stroke. Crit Care Med 37 (7 Suppl): S243-S249, 2009.

9. Liu W, Wang X, Wang Y, Dai Y, Xie Y, Ping Y, Yin B, Yu P, Liu Z, Duan X, et al: SGK1 inhibition-induced autophagy impairs prostate cancer metastasis by reversing EMT. J Exp Clin Cancer Res 37: 73, 2018.

10. Mason JA and Schafer ZT: SGK1 and PHLPP1: Ras-mediated effectors during ECM-detachment. Cell Cycle 15: 2233-2234, 2016.

11. Zhu M, Wu G, Li YX, Stevens JK, Fan CX, Spang A and Dong MQ: Serum- and Glucocorticoid-Inducible Kinase-1 (SGK-1) plays a role in membrane trafficking in caenorhabditis elegans. PLoS One 10: e0130778, 2015.

12. Zuleger T, Heinzelbecker J, Takacs Z, Hunter C, Voelk1 J, Lang F and Proikas-Cezanne T: SGK1 Inhibits autophagy in murine muscle tissue. Oxid Med Cell Longev 2018: 4043726, 2018

13. Nishida Y, Nagata T, Takahashi Y, Sugahara-Kobayashi M, Murata A and Asai S: Alteration of serum/glucocorticoid regulated kinase-1 (sgk-1) gene expression in rat hippocampus after transient global ischemia. Brain Res Mol Brain Res 123: 121-125, 2004.

14. Zhang W, Qian CY and Li SQ: Protective effect of SGK1 in rat hippocampal neurons subjected to ischemia reperfusion. Cell Physiol Biochem 34: 299-312, 2014

15. Cong B, Du J, Zhu X, Lu J and Ni X: Estrogen enhancement of SGK1 expression induced by urocortin contributes to its cardioprotection against ischemia/reperfusion insult. Int J Cardiol 178: 200-202, 2015

16. Ashkenazi A, Bento CF, Ricketts T, Vicinanza M, Siddiqi F, Pavel M, Squitieri F, Hardenberg MC, Imarisio S, Menzies FM and Rubinsztein DC: Polyglutamine tracts regulate beclin 1-dependent autophagy. Nature 545: 108-111, 2017.

17. Lu J, Qian HY, Liu LJ, Zhou BC, Xiao Y, Mao JN, An GY, Rui MZ, Wang T and Zhu CL: Mild hypothermia alleviates excessive autophagy and mitophagy in a rat model of asphyxial cardiac arrest. Neurol Sci 35: 1691-1699, 2014

18. Wang J, Pan XL, Ding LJ, Liu DY, Da-Peng Lei and Jin T: Aberrant expression of Beclin-1 and LC3 correlates with poor prognosis of human hypopharyngeal squamous cell carcinoma. PLoS One 8: e69038, 2013.

19. Schmitz KJ, Ademi C, Bertram S, Schmid KW and Baba HA Prognostic relevance of autophagy-related markers LC3, p62/sequestosome 1, Beclin-1 and ULK1 in colorectal cancer patients with respect to KRAS mutational status. World J Surg Oncol 14: 189, 2014

20. Zhang L, Niu W, He Z, Zhang Q, Wu Y, Jiang C, Tang C, Hu Y and Jia J: Autophagy suppression by exercise pretreatment and p38 inhibition is neuroprotective in cerebral ischemia. Brain Res 1587: 127-132, 2014

21. Zhang X, Yan H, Yuan Y, Gao J, Shen Z, Cheng Y, Shen Y, Wang RR, Wang X, Hu WW, et al: Cerebral ischemia-reperfusion-induced autophagy protects against neuronal injury by mitochondrial clearance. Autophagy 9: 1321-1333, 2013.

22. Vahidinia Z, Alipour N, Atlasi MA, Naderian H, Beyer C and Azami Tameh A: Gonadal steroids block the calpain-1-dependent intrinsic pathway of apoptosis in an experimental rat stroke model. Neurol Res 39: 54-64, 2017.

23. Seyedemadi P, Rahnema M, Bigdeli MR, Oryan S and Rafati H: The neuroprotective effect of rosemary (Rosmarinus officinalis L.) Hydro-alcoholic extract on cerebral ischemic tolerance in experimental stroke. Iran J Pharm Res 15: 875-883, 2016.
24. Harawa V, Njie M, Kessler A, Choko A, Kumwenda B, Kampondeni S, Potchen M, Kim K, Jaworowski A, Taylor T, et al: Brain swelling is independent of peripheral plasma cytokine levels in Malawian children with cerebral malaria. Malar J 17: 435, 2018.

25. Lazzaro MA and Prabhakaran S: Induced hypothermia in acute ischemic stroke. Expert Opin Investig Drugs 17: 1161-1174, 2008.

26. Jickling GC and Sharp FR: Improving the translation of animal ischemic stroke studies to humans. Metab Brain Dis 30: 461-417, 2015.

27. Lehman LL and Rivkin MJ: Perinatal arterial ischemic stroke: Presentation, risk factors, evaluation, and outcome. Pediatr Neurol 51: 760-768, 2014

28. Andresen M, Gazmuri JT, Marín A, Regueira T and Rovegno M: Therapeutic hypothermia for acute brain injuries. Scand J Trauma Resusc Emerg Med 23: 42, 2015.

29. Crossley S, Reid J, McLatchie R, Hayton J, Clark C, MacDougall $\mathrm{M}$ and Andrews PJ: A systematic review of therapeutic hypothermia for adult patients following traumatic brain injury. Crit Care 18: R75, 2014.

30. Neimark MA, Konstas AA, Choi JH, Laine AF and Pile-Spellman J: The role of intracarotid cold saline infusion on a theoretical brain model incorporating the circle of willis and cerebral venous return. Conf Proc IEEE Eng Med Biol Soc 2007: 1140-1143, 2007.

31. Neimark MA, Konstas AA, Lee L, Laine AF, Pile-Spellman J and Choi J: Brain temperature changes during selective cooling with endovascular intracarotid cold saline infusion: Simulation using human data fitted with an integrated mathematical model. J Neurointerv Surg 5: 165-171, 2013.

32. Jaramillo A, Illanes S and Diaz V: Is hypothermia useful in malignant ischemic stroke? Current status and future perspectives. J Neurol Sci 266: 1-8, 2008.

33. Kollmar R, Blank T, Han JL, Georgiadis D and Schwab S: Different degrees of hypothermia after experimental stroke: Short- and long-term outcome. Stroke 38: 1585-1589, 2007.

34. Rusai K, Prokai A, Juanxing C, Meszaros K, Szalay B, Pásti K, Müller V, Heemann U, Lutz J, Tulassay T and Szabo AJ: Dexamethasone protects from renal ischemia/reperfusion injury: A possible association with SGK-1. Acta Physiol Hung 100: 173-185, 2013.

35. Rusai K, Prókai A, Szebeni B, Mészáros K, Fekete A, Szalay B, Vannay Á, Degrell P, Müller V, Tulassay T and Szabó AJ: Gender differences in serum and glucocorticoid regulated kinase-1 (SGK-1) expression during renal ischemia/reperfusion injury. Cell Physiol Biochem 27: 727-738, 2011

36. Yang X, Wu X, Wu K, Yang D, Li Y, Shi J and Liu Y: Correlation of serum- and glucocorticoid-regulated kinase 1 expression with ischemia-reperfusion injury after heart transplantation. Pediatr Transplant 19: 196-205, 2015.

37. Baban B, Liu JY and Mozaffari MS: SGK1 regulates inflammation and cell death in the ischemic-reperfused heart: Pressure-related effects. Am J Hypertens 27: 846-856, 2014.

38. Chiang WC, Wei Y, Kuo YC, Wei S, Zhou A, Zou Z, Yehl J, Ranaghan MJ, Skepner A, Bittker JA, et al: High-throughput screens to identify autophagy inducers that function by disrupting Beclin 1/Bcl-2 binding. ACS Chem Biol 13: 2247-2260, 2018.

39. Jin Y, Lin Y, Feng JF, Jia F, Gao GY and Jiang JY: Moderate hypothermia significantly decreases hippocampal cell death involving autophagy pathway after moderate traumatic brain injury. J Neurotrauma 32: 1090-1100, 2015.

40. Gewirtz DA: The four faces of autophagy: Implications for cancer therapy. Cancer Res 74: 647-651, 2014.

41. Kroemer G: Autophagy: A druggable process that is deregulated in aging and human disease. J Clin Invest 125: 1-4, 2015.

42. Parzych KR and Klionsky DJ: An overview of autophagy: Morphology, mechanism, and regulation. Antioxid Redox Signal 20: 460-473, 2014

43. White E, Mehnert JM and Chan CS: Autophagy, metabolism, and cancer. Clin Cancer Res 21: 5037-5046, 2015.

44. Chen Y, Li X, Wu X, He C, Guo L, Zhang S, Xiao Y, Guo W and Tan B: Autophagy-related proteins LC3 and Beclin-1 impact the efficacy of chemoradiation on esophageal squamous cell carcinoma. Pathol Res Pract 209: 562-567, 2013.

45. Ezzeldin M, Borrego-Diaz E, Taha M, Esfandyari T, Wise AL, Peng W, Rouyanian A, Asvadi Kermani A, Soleimani M, Patrad E, et al: RalA signaling pathway as a therapeutic target in hepatocellular carcinoma (HCC). Mol Oncol 8: 1043-1053, 2014. 